\title{
Educación virtual en imagenología aplicada a la terapia: ¿barrera u oportunidad de mejora?
}

Virtual education in medical imaging applied to therapy: barrier or opportunity for improvement?

\author{
Walter J. Meza Salas ${ }^{1}$, Carlos M. Escobar Galindo ${ }^{1}$
}

\section{RESUMEN}

Objetivo: Determinar si hubo diferencia en las calificaciones del curso de imagenología aplicada a la terapia en la modalidad de educación virtual y presencial en estudiantes de pregrado de una universidad en Lima. Material y Métodos: Estudio descriptivo correlacional. Se comparó dos grupos de estudiantes uno que cursaba la asignatura en modalidad virtual y otro en modalidad presencial del curso de imagenología aplicada a la terapia y se determinó la diferencia entre ellos. Se aplicó estadísticas de diferencias de medias ( T student) y pruebas de normalidad (K-S). Resultados: La muestra estuvo conformada por 64 estudiantes con edad promedio de $21(1,5)$ años y con predominancia de mujeres $(81,25 \%)$. De los cuales $42,2 \%$ desarrollaron el curso en la modalidad presencial durante el año 2019 y $57,8 \%$ de forma virtual en el año 2020. Se demostró que existe una diferencia significativa de las calificaciones de evaluación conceptual $(\mathrm{p}<0,01)$, imagenológica $(\mathrm{p}<0,01)$, tarea académica $(\mathrm{p}<0,01)$ y final $(\mathrm{p}<0,01)$. Además,se evidencio una diferencia significativa de la calificación final en estudiantes del sexo femenino $(\mathrm{p}<0,05)$. Las calificaciones y la edad no estuvieron asociadas en ninguna modalidad de estudios $(\mathrm{p}>0,05)$. Conclusiones: se evidencia diferencia significativa de las calificaciones del curso de imagenología aplicada a la terapia entre las modalidades descritas, siendo mayor en la modalidad virtual .

PALABRAS CLAVE: Diagnóstico por imágenes, rehabilitación, educación a distancia, radiología.

\section{SUMMARY}

Objective: To determine if there was a difference in the grades of the subject medical imaging applied to therapy in the virtual and face-to-face education modality in undergraduate students of a university in Lima. Material and Methods: Descriptive correlational study. Two groups of students were compared, one taking the course in virtual mode and the other in the face-to-face mode of the medical imaging to therapy subject to determine the difference in final grades. Mean difference statistics (T student) and normality tests (K-S) were applied. Results: The sample consisted of 64 students with a mean age of 21 (1.5) with a predominance of women (81.25\%). Of which $42.2 \%$ developed the course in the face-to-face modality during the year 2019 and $57.8 \%$ virtually in the year 2020. It was demonstrated that there is a significant difference in the grades of conceptual evaluation $(p<0.01)$, imaging $(p<0.01)$, academic task $(\mathrm{p}<0.01)$ and final $(\mathrm{p}<0.01)$. In addition, there was a significant difference in the final grade in female students $(p<0.05)$. Grades and age were not associated in any study modality $(p>0.05)$. Conclusion: There was evidence of a significant difference in the grades of the medical imaging applied to therapy subject between the described modalities, being higher in the virtual modality.

KEY WORDS: Diagnostic imaging, rehabilitation, education, distance, radiology.

\footnotetext{
Facultad de Medicina, Universidad Peruana Cayetano Heredia. Lima, Perú.
} 


\section{INTRODUCCIÓN}

En la actualidad, el mundo se encuentra inmerso en una pandemia causada por la COVID-19 que ha obligado a declarar en estado emergencia la educación en todos sus niveles. Ante dicha problemática las instituciones educativas, docentes y estudiantes estuvieron forzados a abandonar al 100\% la modalidad presencial para evitar la contaminación cruzada y respetar el distanciamiento social impartido por las autoridades $(1,2)$.

Esto constituye un gran desafío para sacar adelante el sistema educativo, debido a que tradicionalmente se impartía educación presencial y muchas casas de estudio no estaban preparadas para la modalidad cien por ciento virtual en gran medida debido al precario acceso a internet (3). Este cambio abrupto no permitió la oportuna capacitación de los estudiantes y docentes para afrontar la necesidad imperante de continuar con la construcción de conocimientos y logro de competencias (1). Sin embargo, es también verdad que la educación virtual ha ido progresando lentamente en el país estableciendo inclusive programas semipresenciales de posgrado con al menos 50\% de virtualidad en donde se han obtenido resultados positivos (4).

A pesar de reducir la brecha de acceso a la educación superior en tiempo y espacio, el prejuicio y rechazo existente hacia la educación virtual crea la falsa idea de que es una modalidad impersonal. Sin embargo, si el docente aplicara correctamente las Tecnologías de la Información y la Comunicación (TICs), y tuviese una buena actitud hacia el estudiante podría asumir un compromiso más activo que permita una mayor retroalimentación y flexibilización en el proceso de humanización del aprendizaje virtual $(5,6)$.

Los antecedentes respecto a la educación virtual y su relación con las calificaciones aún son limitados especialmente en cursos que demandan un aprendizaje más visuoespacial y de apoyo al diagnóstico médico como es el análisis imagenológico. El análisis de imágenes de ayuda al diagnóstico e intervención terapéutica demanda que los estudiantes tengan que identificar, discriminar, estructuras anatómicas y las alteraciones de estas a través de representaciones bidimensionales y multiplanares. En situaciones de presencialidad el docente hace uso de imágenes físicas y útiles de escritorio para el desarrollo de su clase. Situación que ha cambiado gracias a la virtualización que ofrece recursos distintos a los de la modalidad presencial que podrían facilitar este proceso tales como flexibilidad, seguimiento sincrónico, asincrónico y TICs $(5,7,8)$.

Todos estos cambios han llegado a modificar y replantearse la forma de enseñar y aprender, quedándose en el ámbito educativo como parte de la "nueva normalidad" la cual está sujeto a los avances tecnológicos. Por estas razones, el presente artículo pretende establecer un punto de partida en la mejora del aprendizaje virtual de imagenología aplicada a estudiantes de terapia física y ocupacional tomando en cuenta el uso de recursos virtuales.

El presente estudio tuvo como objetivo determinar si hubo diferencia en las calificaciones del curso de imagenología aplicada a la terapia en la modalidad de educación virtual y presencial en estudiantes de pregrado.

\section{MATERIAL Y MÉTODOS}

El estudio fue de tipo descriptivo, correlacional y retrospectivo. La muestra estuvo conformada por estudiantes de la carrera de Tecnología Médica en terapia física y ocupacional del quinto ciclo que se encontraban cursando la asignatura de Imagenología Aplicada a la terapia en una Universidad Peruana. Se excluyeron a los estudiantes que no siguieron el curso regularmente o se retiraron.

\section{Procedimiento}

El curso de Imagenología aplicada a la terapia fue de naturaleza teórica, y tuvo como objetivo familiarizar al estudiante con la semiología radiológica visceral y del aparato locomotor, para comprender de forma integral, describir y diferenciar la anatomía y patología radiológica en imágenes bidimensionales y multiplanares de ayuda al diagnóstico idóneas para su representación.

El curso tuvo una duración de 16 semanas tanto en el año 2019 como 2020. Consta de una evaluación integral ponderada $(100 \%)$ dividida en tres aspectos: el "conceptual" (30\%), "imagenológico" (50\%) y "tarea académica" (20\%). La Evaluación "conceptual" representa al manejo de información (definiciones, terminología, teorías, principios y hechos), mientras que la "imagenológica" consistió en el reconocimiento y descripción de la anatomía y patología radiológica representada en imágenes de ayuda al diagnóstico. Ambos aspectos fueron evaluados mediante tres 
exámenes virtuales $\mathrm{o}$ presenciales dependiendo de la modalidad disponible. Finalmente, la "tarea académica" estuvo conformada por tres trabajos individuales en los cuales el estudiante debía aplicar sus conocimientos conceptuales e imagenológicos según su juicio crítico.

El curso virtual se desarrolló en el año 2020, mientras que el presencial se realizó en los salones de la universidad durante el periodo 2019. Los cursos fueron llevados en un horario similar, el mismo contenido y sistema de evaluación.

Se recolectaron las calificaciones parciales y finales de los diferentes aspectos de evaluación con el objetivo de ser comparados guardando la confidencialidad de los participantes.

\section{Análisis de datos}

Los datos fueron analizados mediante el programa estadístico SPSS versión 25.0. Se determinó las medidas de tendencia central y dispersión para las calificaciones obtenidas y edad. Para determinar la normalidad de los datos se realizo la prueba K-S [Kolmogórov-Smirnov]. Se utilizó la Prueba de Pearson para correlacionar las calificaciones y la edad, considerándose significativos valores menores al error estándar $(\mathrm{p}<0,05)$
Se aplico La prueba $\mathrm{T}$ de Student para grupos independientes para contrastar las calificaciones en función de las variables de agrupación denominada "modalidad" y "sexo". Considerándose estadísticamente significativos valores menores a 0,05 $(\mathrm{p}<0,05)$.

\section{RESULTADOS}

En total participaron 64 estudiantes, 12 varones $(18,75 \%)$ y 52 mujeres $(81,25 \%)$ de una edad promedio de 20,9 $(1,5)$ años, siendo la edad mínima de 19 años y la máxima de 26 años. Cabe resaltar que 27 de ellos desarrollaron el curso de Imagenología Aplicada a la terapia en la modalidad presencial $(42,2 \%)$ durante el año 2019 , y 37 de forma virtual $(57,8 \%)$ en el año 2020 durante la actual pandemia causada la COVID-19.

Se evidenció una diferencia significativa en las calificaciones de evaluación conceptual $(\mathrm{t}=-6,54$, $\mathrm{p}<0,01)$, imagenológica $(\mathrm{t}=-4,58, \mathrm{p}<0,01)$, tarea académica $(\mathrm{t}=-4,09, \mathrm{p}<0,01)$ y final $(\mathrm{t}=-6,35, \mathrm{p}<0,01)$ del curso de Imagenología en la modalidad virtual versus presencial. Las calificaciones en modalidad presencial fueron menores a las virtuales. Además, los resultados presentaron un tamaño de efecto grande $(\mathrm{d}$ cohen $>=1$ ) (tabla 1).

Tabla 1. Comparación y diferencia de calificaciones del curso de imagenología en educación virtual y presencial

\begin{tabular}{llllllll}
\hline \multirow{2}{*}{ Evaluación } & \multicolumn{2}{l}{ Presencial } & \multicolumn{2}{l}{ Virtual } & & \multicolumn{2}{l}{} \\
& $\mathbf{n}$ & $\mathbf{M}(\mathbf{s})$ & $\mathbf{n}$ & $\mathbf{M}(\mathbf{s})$ & $\boldsymbol{p}$ & $\mathbf{t}$ & d de Cohen \\
\hline Conceptual & 27 & $15,2(1,8)$ & 37 & $17,9(1,5)$ & 0,01 & $-6,54$ & 1,6 \\
Imagenológica & 27 & $15,5(1,9)$ & 37 & $17,8(2,0)$ & 0,01 & $-4,58$ & 1,1 \\
Tarea académica & 27 & $15,3(1,4)$ & 37 & $16,9(1,7)$ & 0,01 & $-4,09$ & 1 \\
Final & 27 & $15,4(1,4)$ & 37 & $17,6(1,5)$ & 0,01 & $-6,35$ & 1,6 \\
\hline
\end{tabular}

Nota: $\mathrm{p}<0,05$ diferencia significativa; $\mathrm{n}=$ muestra; M: Media ; $\mathrm{s}$ desviación estándar; $\mathrm{T}=\mathrm{T}$-Student para muestras independientes

Tabla 2. Comparación y diferencia de calificación en Evaluación Final del curso de imagenología modalidad virtual versus presencial, según sexo

\begin{tabular}{llllllll}
\hline & \multicolumn{2}{c}{ Presencial } & \multicolumn{3}{c}{ Virtual } & & \\
Sexo & $\mathbf{n}$ & $\mathbf{M}(\mathbf{s})$ & $\mathbf{n}$ & $\mathbf{M}(\mathbf{s})$ & $\boldsymbol{p}$ & $\mathbf{t}$ & d de Cohen \\
\hline masculino & 8 & $15,1(0,7)$ & 4 & $16,3(2)$ & 0,32 & $-1,17$ & 0,7 \\
femenino & 19 & $15,6(1,5)$ & 33 & $17,9(1,3)$ & 0,01 & $-5,52$ & 1,6
\end{tabular}

Nota: $\mathrm{p}<0.05$ diferencia significativa; $\mathrm{n}=$ muestra; $\mathrm{M}$ : Media ; $\mathrm{s}$ : desviación estándar; $\mathrm{T}=\mathrm{T}-$ Student para muestras independientes 
Con respecto al sexo de los participantes se evidenció una diferencia significativa en la calificación final del curso en estudiantes del sexo femenino $(t=-5,52, p<0,05)$, mas no en el masculino $(\mathrm{t}=-1,17, \mathrm{p}=0,32)$. La calificación final en la modalidad presencial fue menor a la virtual en el sexo femenino, con un tamaño del efecto grande ( $\mathrm{d}$ cohen=1,6). (tabla 2)

Las calificaciones y la edad no estuvieron asociadas en ninguna modalidad de estudios $(\mathrm{p}>0,05)$

\section{DISCUSIÓN}

Los resultados del estudio señalaron un mayor puntaje en las calificaciones del curso de Imagenología aplicada a terapia en la modalidad de educación virtual en comparación a la presencial, lo cual podría deberse a múltiples factores como la retroalimentación, tutoría, didáctica, flexibilidad y el sistema educativo de la universidad.

La educación virtual en imagenología a diferencia de la presencial ofrece además del desarrollo sincrónico de la clase, la posibilidad de su posterior revisión asincrónica mediante videos de imágenes y del proceso de obtención de ellas o presentaciones de power point con audio. También, permitió la generación de foros asincrónicos de discusión de casos clínicos basados en imágenes bidimensionales y multiplanares de ayuda al diagnóstico que facilitaron la retroalimentación y seguimiento continuo del proceso de construcción de conocimientos humanizando el proceso de aprendizaje virtual $(5,6)$.

En la actualidad los docentes asumen un rol más activo como tutores o guías en el aprendizaje colaborativo a diferencia del paradigma tradicional en la que se impartía conocimiento de manera vertical y poco constructiva (8). Gracias a la virtualización, los estudiantes tienen mayores fuentes de información a la mano para poder afirmar, refutar y aportar a la construcción de conocimientos y entablar una relación más dinámica con sus docentes.

El docente en educación virtual debe elaborar material didáctico que facilite el aprendizaje, además de ser más empático, demostrar entusiasmo, ser compresivo y flexible con sus estudiantes, más aún durante la actual pandemia (9-11). A pesar de las limitaciones tecnológicas, este cambio en la didáctica puede llegar a ser mas inmersivo en los estudiantes mejorando la relación con sus docentes. Sin embargo, debería estar plasmado también dentro de las cargas docente las horas virtuales no lectivas debido a que el proceso de transformación de material educativo presencial a virtual demanda de mayor esfuerzo mental y cognitivo. Si bien estos procesos pueden ser beneficiosos para los estudiantes, para los docentes pueden llegar a ser muy desafiantes. La transformación de imágenes radiológicas a formatos digitales visibles y valorables listos para la enseñanza demanda una mayor carga mental a los docentes.

La disponibilidad de los recursos tecnológicos y la calidad de la información brindada es vital para afrontar con éxito el reto de la virtualización de la educación superior $(12,13)$. Si la universidad contará con suficientes tecnologías de la información y comunicación podría derribar con mayor efectividad las barreras de tiempo y espacio, generando que la relación estudiante - docente sea más activa y se fomente el aprendizaje colaborativo.Sin embargo, la utilización de tecnología depende en gran medida del acceso a internet. En el Perú la conexión a internet es deficiente y limitada no siendo homogénea a lo largo de su territorio por lo que puede ser una limitante directa que influya sobre el proceso de aprendizaje virtual de los estudiantes $(2,3)$.

Diversos estudios realizados en estudiantes universitarios afirman que las mujeres presentan mejores habilidades sociales, emocionales y cognitivas que conllevan al éxito en su rendimiento académico (14-17). Esto podría explicar porque las estudiantes mujeres en el estudio respondieron y se adaptaron mejor a la educación virtual que los varones, lo cual se ve reflejado en la calificación final. Por otro lado, los varones desarrollan mejor capacidad matemática y visoespacial mientras que las mujeres destacan en habilidades verbales y de memoria por lo que pondría en discusión los resultados $(18,19)$. Además, pudo influir el gran número de mujeres en la muestra, sin embargo podría ser motivo de discusión para futuros estudios.

La actual pandemia causada por la COVID-19 ha forzado tanto a los estudiantes como a los docentes a adaptarse a la virtualidad, mejorando las estrategias de aprendizaje e interacción estudiante-docente (1). A pesar que la pandemia ha traído consecuencias nefastas a los sistemas de salud constituyéndose como una amenaza directa al orden mundial, también fue una oportunidad para demostrar como los sistemas educativos universitarios pueden adaptarse a la nueva normalidad utilizando los recursos virtuales que 
permitan un mejor aprovechamiento de las imágenes digitales de ayuda al diagnóstico e intervención terapéutica .

Es un hecho que la educación virtual no reemplaza a la presencial, sin embargo, a pesar de que ofrece mayores recursos de accesibilidad sobre todo en tiempo y espacio es frecuente la deserción de los usuarios (20,21). Para evitarlo, es importante conocer las necesidades e inquietudes de los estudiantes y adoptar una actitud más proactiva y comprensiva que motive su proceso de aprendizaje. Aunque es un punto relevante, en el estudio no se presentaron deserciones de los estudiantes.

Como principales retos a futuro se debería considerar la posibilidad de crear entornos simulados a través de la realidad virtual que facilite la inmersión de los estudiantes en un proceso real de análisis de imágenes médicas radiológicas relacionándolo con la anatomía y patologías en tiempo real. Estos sistemas pueden ser una oportunidad de motivación intrínseca y de mayor apertura para un aprendizaje activo y sistémico.

Entre las principales limitaciones del estudio se puede mencionar la falta de acceso a otras características personales (habilidades sociales, emocionales y cognitivas) de los estudiantes que pudieron estar involucradas en la obtención de las calificaciones bajas.

El presente artículo constituye un primer paso para abrir camino a futuras investigaciones no solo en el ámbito de la imagenología sino a diferentes asignaturas que permitan comparar la modalidad presencial, virtual y la híbrida que está a puertas de desarrollarse durante el año 2022 en el Perú. La transformación del modelo educativo tradicional (presencial) en conjunto con la educación virtual se conoce como aprendizaje híbrido, el cual toma fuerza durante la actual pandemia como una alternativa para el regreso a la presencialidad respetando el marco normativo y asegurando una educación de calidad $(22,23)$

\section{Correspondencia}

Walter J. Meza Salas

Correo elecronico: walter.meza@upch.pe

Declaración de autores: Los autores declaran no tener ningún conflicto de interés en el presente trabajo de investigación.

\section{REFERENCIAS BIBLIOGRÁFICAS}

1. Lovón Cueva MA, Cisneros Terrones SA. Repercusiones de las clases virtuales en los estudiantes universitarios en el contexto de la cuarentena por COVID-19: El caso de la PUCP. Propósitos Represent. 2020; 8. DOI: 10.20511/pyr2020.v8nSPE3.588

2. Huanca-Arohuanca J, Supo-Condori F, Leon RS, Quispe LS. El problema social de la educación virtual universitaria en tiempos de pandemia, Perú. Innovaciones Educ. 2020;22(Especial):115-28.

3. Flores-Cueto JJ, Hernández RM, Garay-Argandoña R. Tecnologías de información: Acceso a internet y brecha digital en Perú. Rev Venez Gerenc. 2020;25(90):504-27.

4. Huamán D, García RB, Tacilla J. La calidad educativa virtual en estudiantes de la modalidad semi presencial en el Perú: Una revisión sistemática.Tesis de Bachiller. Lima, Péru: Universidad Peruana Unión; 2020.

5. Sepúlveda-Romero ME. Humanización del acto de la retroalimentación en la educación virtual. RevistaVirtu@Imente. 2019; 7(1): 95-115. DOI: 10.21158/2357514x.v7.n1.2019.2326

6. Lozada O, Beltrán O, Vargas F, Martín D, Hincapié $\mathrm{B}$, Herrera $\mathrm{M}$, et al. Humanización de la práctica docente universitaria. Tesis para titulo de de especialista en pedagogía. Bogota, Colombia: Universidad de San Buenaventura; 2013.

7. Garrido F. Una invitación a repensar la enseñanza en radiología. Rev Chil Radiol. 2020; 26(3):86-7.

8. Quiroz JS. El rol del tutor en los entornos virtuales de aprendizaje. Innov Educ. 2010;10(52):13-23.

9. Alonso L, Blázquez F. El docente de educación virtual. Guía básica: incluye orientaciones y ejemplos del uso educativo de Moodle. Madrid: Narcea Ediciones; 2016.

10. Novoa A, Pirela J. Accompaniment from an ethics of life to educate in times of pandemic. Utop Prax Latinoam. 2020;25(Extra4):11.

11. Salinas-Ibáñez J. Entornos virtuales y formación flexible. Rev Tecnol En Marcha. 2004;17(3):55-69.

12. Gagliardi V. Desafíos educativos en tiempos de pandemia. Questión. 2020;1: e312. DOI: $10.24215 / 16696581 \mathrm{e} 312$

13. Llopiz Guerra K, Gómez NA, González Peña R, Alberca NE, Fuster-Guillén D, Palacios-Garay J. Prácticas educativas inclusivas a través de la educación a distancia. Experiencias en Cuba. Propósitos Represent. 2020;8(2).

14. Bandeira M, Rocha SS, Freitas LC, Del Prette ZAP, Del Prette A. Habilidades sociais e variáveis sociodemográficas em estudantes do ensino fundamental. Psicol Em Estudo. 2006;11:541-9.

15. Iturra GO, Astete EP, Jara MO. Habilidades sociales y rendimiento académico: una mirada desde el 
género. Acta Colomb Psicol. 2012;15(2):21-8.

16. Echavarri M, Godoy JC, Olaz F. Diferencias de género en habilidades cognitivas y rendimiento académico en estudiantes universitarios. Univ Psychol. 2007;6 (2):319-29.

17. Robbins SB, Allen J, Casillas A, Peterson CH, Le $H$. Unraveling the differential effects of motivational and skills, social, and self-management measures from traditional predictors of college outcomes. J Educ Psychol. 2006;98(3):598.

18. Halpern DF. Sex differences in cognitive abilities. East Sussex, United Kingdom: Psychology press; 2000.

19. Pérez A, Mammarella I, Del Prete F, Bajo T, Cornoldi C. Capacidad geométrica y memoria visoespacial en población adulta. Psicológica. 2014;35(2):225-49.

20. La Madriz J. Factores que promueven la deserción del aula virtual. Orb Rev Científica Cienc Humanas. 2016;12(35):18-40.
21. Lopez EG, Chiyong IE. Rendimiento académico y deserción de estudiantes universitarios de un curso en modalidad virtual y presencial. RIED Rev Iberoam Educ Distancia. 2021;24(2):189-202.

22. Chavez REG, Cobeña ÁWV, Hidalgo ERC. La Educación Híbrida como alternativa frente al Covid-19 en el Ecuador: Hybrid education as an alternative to Covid-19 in Ecuador. Tse'De. 2020;3(1): 0-0.

23. Viñas M. Retos y posibilidades de la educación híbrida en tiempos de pandemia. Plurentes. Artes Y Letras. 2021; 12: 027. Doi: 10.24215/18536212e027

Recibido: 24/07/2020

Aceptado: 14/11/2020 